\title{
Erster Teil, zweites Buch: Die Welt und ihr Sinn oder Metalogik
}

\author{
Emanuele Pompetti
}

\section{Einführung}

In diesem Aufsatz wird das zweite Buch des ersten Teils des Stern der Erlösung (GS II, S. 44-66) von Franz Rosenzweig dargestellt. Der rote Faden der Darstellung wird der Begriff der metalogischen Welt sein und dies aus mehreren Gründen. Einerseits trägt der Teil, der hier behandelt wird, eben diese Überschrift: «Die Welt und ihr Sinn oder Metalogik» $»^{52}$. Andererseits ist anzumerken, dass der gesamte Stern metalogisch aufgebaut ist.

Was dies bedeutet, soll im Folgenden dargestellt werden. Dafür ist allerdings ein Ausgangspunkt nötig und dieser kann nur der sein, der am Anfang des Sternes von Rosenzweig selbst vertreten wird: der Tod bzw. die Angst vor dem Tod. Rosenzweig tut dies, weil der Tod eine bestimmte Beziehung zum Nichts, also zum Punkt Null der Erkenntnis, darstellt ${ }^{53}$, und Rosenzweig schreibt:

52 GS II, 44.

${ }^{53}$ Rosenzweig schreibt: «Das Nichts ist nicht Nichts, es ist Etwas. Im dunkeln Hintergrund der Welt stehen als ihre unerschöpfliche Voraussetzung tausend Tode, statt des einen Nichts, das wirklich Nichts wäre, tausend Nichtse, die, eben weil viele, Etwas sind.» (GS II, 5; kursiv von mir). Der Tod hat also

How to cite this book chapter:

Pompetti, E. 2021. Erster Teil, zweites Buch: Die Welt und ihr Sinn oder Metalogik. In: Brasser, M., Bojanić, P. and Ciglia, F. P. (eds.) The Star for Beginners: Introductions to the Magnum Opus of Franz Rosenzweig. Pp. 41-54. London: Ubiquity Press. DOI: https://doi.org/10.5334/bco.e. License: CC-BY 
«Von den Nichtsen des Wissens stösst unsere Entdeckerfahrt vor zum Etwas des Wissens.» ${ }^{54}$

Das Nichts als Ausgangspunkt der Erkenntnis über den Sinn der Welt wird sich in zwei Wege spalten, die aber komplementär sind und die jeweils auf verschiedene Erkenntnisse bringen werden. Diese zwei Wege werden von Rosenzweig «de[r] Weg der Bejahung dessen, was nicht Nichts ist, und de[r] Weg der Verneinung des Nichts ${ }^{55}$ genannt. Hier wird nun der Weg vom Tode über das Nichts bis zur metalogischen Welt im Stern rekonstruiert.

Bevor mit dem Eigentlichen begonnen wird, ist eine kleine Anmerkung nötig. In diesem Aufsatz ist der vielleicht etwas einfache Stil der Darstellung zu entschuldigen, der aber, dem Ziel dieses Bandes dienend, Klarheit in der Darstellung schaffen möchte. Aus diesem Grund und aus Gründen des eingeschränkten Umfangs dieses Aufsatzes sind die Verweise, die der erfahrene Philosoph sicherlich vermissen wird, auf andere Philosophen und deren Werke bzw. auf Sekundärliteratur vermieden bzw. massiv eingeschränkt.

\section{Vom Tode}

«Vom Tode, von der Furcht des Todes, hebt alles Erkennen des All an» ${ }^{56}$. Wir können versuchen, mit Rosenzweig diesen Weg zu gehen: aus der Angst des Todes, Erkenntnis über die Welt zu gewinnen.

Was ist der Tod? Wir wissen wahrscheinlich wenig darüber, ja vielleicht sogar nichts. Aber heisst das, dass der Tod nichts ist? Nein, nur wir wissen nichts darüber. Wir fürchten den Tod wahrscheinlich deswegen, weil wir nichts über ihn wissen und weil er uns auf jeden Fall angeht: nur eins ist sicher, wir werden irgendwann sterben. Diese Wahrheit, die bei Rosenzweig durch so etwas wie eine Urangst, die jeder hat, bewusst oder unbewusst, ans Licht kommt, kann keine Lehre verleugnen. Man mag dem Tod jegliche Bedeutung geben (ein Scheintod, ein Gehen ins Jenseits, ein Auseinandergehen der Moleküle, aus denen wir bestehen, ein gehen ins Nichts, usw.), sicher aber ist nur eins: jeder, alles muss einst sterben. Wenn der Tod dermassen beängstigt, dass er dazu drängt, nach Erkenntnis zu suchen, um sich zu beruhigen, weil niemand in und mit dieser Spannung ein schönes Leben verbringen kann, wenn der Tod die unausweichliche Frage ist, können wir nicht behaupten, dass er nichts sei. Also ist der Tod etwas. Aber Was?

eine Beziehung zum Nichts. Allerdings ist das Nichts im Stern nicht nur der „Punkt Null“ der Erkenntnis. Hier können wir die anderen Aspekte des Nichts im Stern nicht vertiefen, deswegen verweisen wir auf: L. Bertolino, Il nulla e la filosofia. Idealismo critico ed esperienza religiosa in Franz Rosenzweig, Torino 2005.

${ }^{54}$ GS II, 22.

55 GS II, 23.

56 GS II, 3. 
Der Tod, hier von Rosenzweig als Ausgangspunkt unseres Wissens genommen, ist ein Nichts: das Nichts unseres Wissens und gerade dieses Nichts -Wissen über den Tod, der uns aber unmittelbar angeht, macht uns Angst und drängt uns zur Suche nach der Erkenntnis, denn das, was uns geschieht und das wir nicht verstehen, beängstigt uns. Ausserdem ist mit dieser Urangst ein gewisses Gefühl des Untergehens verbunden. Denn was bedeutet, sich vor dem Tod zu fürchten? Es bedeutet das Gefühl des Untergehens zu haben, sich auch für einen kurzen Moment panisch die Fragen zu stellen: „Sterbe ich?“, „Was passiert im Augenblick des Todes? Und danach?“, „Gehe ich ins Nichts?“, „Wie kann ich dem Tod entgehen? Ist das überhaupt möglich?“, usw. Rosenzweig schreibt dazu: «der Mensch [...] muss sich einmal in seiner furchtbaren Armut, Einsamkeit und Losgerissenheit von aller Welt gefühlt haben und eine Nacht lang Aug in Auge mit dem Nichts gestanden sein. ${ }^{57}$ Allerdings, schreibt Rosenzweig, «ihm [dem Menschen] ist ein [...] Ausweg aus dem Engpass des Nichts bestimmt ${ }^{58}$. Welcher denn? Er schreibt weiter: «Der Mensch soll die Angst des Irdischen nicht von sich werfen; er soll in der Furcht des Todes - bleiben. [...] Die Angst des Irdischen soll von ihm genommen werden nur mit dem Irdischen selbst» ${ }^{59}$.

Wie ist so etwas möglich? Die Philosophie hat Rosenzweigs Meinung nach versucht, die historische Aufgabe zu erfüllen, dem Menschen die Angst vor dem Tod zu nehmen. Wie denn? Wie soll die Philosophie die Angst vor dem Irdischen, vor dem Sterblichen nehmen? Hier finden wir den zentralen, „philosophischen "60 Ansatz Rosenzweigs: "Die Angst des Irdischen soll von ihm genommen werden nur mit dem Irdischen selbst» ${ }^{61}$. Er setzt sich mit der philosophischen Tradition auseinander und nimmt dazu Stellung und seine Lösung, die einzig mögliche für ihn, ist eine metalogische. Im Folgenden werden wir also den Gedankengang Rosenzweigs rekonstruieren, der uns von der Angst des Todes zu einer metalogischen Antwort führen soll.

\section{Der Weg zur Meta-logik}

«Die Angst des Irdischen abzuwerfen, dem Tod seinen Giftstachel, dem Hades seinen Pesthauch zu nehmen, des vermisst sich die Philosophie» ${ }^{62}$. Wie geht

${ }^{57}$ GS II, 4.

58 GS II, 4.

59 GS II, 4.

60 „Philosophisch“ in dem Sinne, dass Rosenzweig hier, im ersten Teil des Sternes, seine Gedanken «in philosophos» (GS II, 3), also „für die Philosophen“ bzw. „im Namen der Philosophen“ formuliert. Denn die Philosophie hat, seiner Meinung nach, nicht das letzte Wort (dies werden wir aber später vertiefen).

${ }^{61}$ GS II, 4; kursiv von mir.

${ }^{62}$ GS II, 3. 
das? Kann die Philosophie das wirklich? Für Rosenzweig ist die Antwort der Philosophie vielleicht unzulänglich, aber es ist sehr wichtig, dass der Mensch diesen Weg geht, denn dies wird ihn dazu bringen, den philosophischen Ansatz zu überwinden und durch die Theologie (zweiter Teil des Sternes - «in theologos» ${ }^{63}$ ) und die Politik (dritter Teil des Sternes - «in tyrannos» ${ }^{64}$ ) hindurch der Weg «[v]om Tode» ${ }^{65}$ (wie es am Anfang des Sternes heisst), «ins Leben» ${ }^{66}$ (wie es am Ende des Sternes heisst) zu gehen. Dieser Weg ist ein fruchtbarer Weg voller Erkenntnis. Denn der Mensch ist ein erkennendes Wesen und diese Erkenntnis hat eine Geschichte, sie entsteht durch einen bestimmten Prozess, den wir hier schildern werden. Ja, das Leben ist auch Erkenntnis, die Erkenntnis ist ein wichtiger Teil unseres Lebens. Der Mensch, der lebt, strebt ein bewusstes Leben an, ein Leben, in dem er weiss, woher er kommt und wohin er geht. Der Mensch möchte wissen, ob er in der Erkenntnis einen Anhaltspunkt haben kann, um sich im Leben zu orientieren. Deswegen muss der Weg zur Erkenntnis beschrieben und geprüft werden. Dies tut Rosenzweig, indem er sich zum „Punkt Null“ des Wissens stellt und daraus versucht, unser Wissen über die drei Elemente, die die Wirklichkeit ausmachen, Gott, Welt und Mensch, $\mathrm{zu}$ rekonstruieren.

Hier ist anzumerken, dass innerhalb des bis jetzt aufgebauten Szenarios bereits alle Elemente der «immerwährenden Vorwelt» ${ }^{67}$ vorhanden sind: Gott, Welt und Mensch. Denn die Frage nach dem Tod setzt ein Wesen, einen Menschen voraus, der lebt (und die Welt besteht aus abertausenden lebendigen Einzelwesen), der sich um sein Leben kümmert (innerhalb eines bestimmten Milieus, der Welt eben, in der der Mensch sich orientieren möchte, ja sogar orientieren muss) und ängstigt, der sich vielleicht sogar die Frage nach einem möglichen Schöpfer des Lebens (Gott) oder Retter stellt (Gott oder der Nächste, der andere Mensch). Diese sind die drei „Nichtse“, über die Rosenzweig im ersten Buch des Sternes nachdenkt. Denn, nachdem ich mir die Frage gestellt habe, ob es Gott, Welt und Mensch gibt, was weiss ich darüber? Erstmal nichts, ich muss erst weiter denken, um Aussagen über sie machen zu können, und das ist eben das, was Rosenzweig in diesem ersten Teil des Sternes macht. Aber auf diesem ersten Niveau (ausgehend vom „Punkt Null der Erkenntnis“) ist in der Frage, was Gott, Mensch und Welt seien, noch keine Antwort enthalten. Keine Antwort, aber schon eine gewisse Orientierung. Gott, Mensch und Welt bezeichnen drei Baustellen, drei Probleme (Gott das Problem der Schöpfung, der Rettung, ja sogar seiner Existenz und das Problem, was er sei - das Problem der Physis Gottes; die Welt das Problem der Lebenden und die Frage der Orientierung des Menschen in ihr, also ob und wie sie verständlich sei - das Problem der Logik;

\footnotetext{
${ }^{63}$ GS II, 103.

${ }^{64}$ GS II, 295.

${ }^{65}$ GS II, 3.

${ }^{66}$ GS II, 472.

67 GS II, 2.
} 
der Mensch das Problem der Rettung und dementsprechend - der Ethik). Hier finden wir eine erste Erklärung der Vorsilbe „meta"68. Rosenzweig schreibt: «Nicht als Gegenstände rationaler Wissenschaft denken wir sie wiederherzustellen, sondern gerade umgekehrt als ,irrationale' Gegenstände. Als Mittel zur ersten Absteckung ihrer Orte diente uns die Methode, die in der Vorsilbe, meta bezeichnet ist» ${ }^{69}$.

\section{Die zwei Wege zum Etwas des Wissens}

Bevor wir mit der Rekonstruktion der Erkenntnis über die Welt (Gott und Mensch werden im ersten und dritten Buch des ersten Teils des Sternes behandelt, deswegen werden sie hier ausgelassen) starten, muss geklärt werden, wie Rosenzweig zu den aufwändigen und etwas dunklen Formulierungen der „Verneinung des Nichts“ und der „Bejahung des Nichtnichts“ kommt.

Angst vor dem Tod zu haben heisst, sich die Frage zu stellen: was ist der Tod? Ist der Tod nichts? - „Nein“, lautet die Antwort. Ist also der Tod etwas? - „Ja“, lautet die Antwort. Was ist aber der Tod? Was weiss ich darüber? - „Nichts.“

Auf diese Weise haben wir schon unsere zwei Wege, von denen Rosenzweig spricht (die „Verneinung des Nichts“ und die „Bejahung des Nichtnichts“) und die uns zur Erkenntnis führen sollten: Ist der Tod nichts? - „Nein.“ Unter dieser Perspektive wird der Tod als „Verneinung des Nichts“ bezeichnet. Ist der Tod etwas? - „Ja.“Was denn? - „Ich weiss es nicht, ich weiss nur, dass er nicht Nichts ist.“ Unter dieser zweiten Perspektive wird der Tod also als „Bejahung des Nichtnichts" bezeichnet.

Unter der ersten Perspektive wird der Tod als nichts verneint (der Tod ist etwas, deshalb kann ich davor Angst haben), aber das bedeutet gleichzeitig ein erster Schritt unseres Wissens über den Tod, ein sich Verabschieden vom Nichts des Wissens über den Tod: kann ich etwas über den Tod behaupten? Kann ich eine positive Aussage über den Tod machen? „Der Tod ist nicht Nichts“ ist eine negative Aussage, also eine „Verneinung des Nichts“ des Todes.

Kann ich dies nun positiv formulieren? Ja: der Tod ist ein „Nichtnichts“. Das „Nichtnichts“ des Todes kann bejaht werden. Wieso sind diese zwei Wege so wichtig und komplementär für Rosenzweig? Weil sie verschiedene Perspektiven eröffnen. Dass der Tod kein Nichts sei, zeigt nur, dass es ein Etwas gibt. Was das denn sei, ist in der Frage nicht enthalten. Eine Verneinung unterbricht das Fragen. Eine Bejahung, eine positive Formulierung, möchte nun mit präzisen

${ }^{68}$ Rosenzweig spricht vom „metaphysischen Gottesbegriff“, vom „metalogischen Weltbegriff“ und vom „metaethischen Menschenbegriff“ (siehe GS II, 20).

${ }^{69}$ GS II, 22. 
Inhalten gefüllt werden. Ich möchte nun nicht nur wissen, ober der Tod nichts sei (Ja-Nein-Frage), sondern auch, was denn der Tod sei. ${ }^{70}$

\subsection{Die „Verneinung des Nichts“}

Wir gehen nun zuerst ${ }^{71}$ den Weg der „Verneinung des Nichts“. Die Welt ist nicht Nichts, sondern etwas. Sie besteht aus einzelnen Wesen, die nicht nichts sind, sondern etwas, so wahr ist es, dass sie auch sterben können. Ist diese eine grosse Erkenntnis? Für Rosenzweig schon, denn somit kritisiert er den Ansatz «der ganzen ehrwürdigen Gesellschaft der Philosophen von Ionien bis Jena» ${ }^{72}$, also von den Griechen bis Hegel, die die Welt «als eine Allheit»" ${ }^{73}$ betrachtet haben. Diese ist die Antwort, für Rosenzweig ja die Lüge, die die Philosophie gepriesen hat, um den Menschen von der Todesfrage zu erleichtern. Rosenzweig schreibt: «die Philosophie leugnet diese Ängste der Erde [...], indem sie den blauen Dunst ihres Allgedankens um das Irdische webt. Denn freilich: ein All würde nicht sterben, und im All stürbe nichts. Sterben kann nur das Einzelne.. ${ }^{74}$ Die Philosophie hat meistens behauptet, dass eigentlich ein All existieren würde (z.B. das Sein bei Parmenides, das Eine bei Plotin, Gott bei Spinoza, der Geist bei Hegel, usw.) und nicht das Einzelne. Das Einzelne wäre nur immer als Offenbarung, als Teil usw. des Alls zu verstehen. Das All hätte ein (wahres) Leben, nicht das Einzelne, und weil das Einzelne nur im All zu verstehen ist und nur in ihm und durch es leben kann, «ist der letzte Schluss dieser Weisheit: der Tod sei - Nichts ${ }^{75}$, denn der Tod des Einzelnen kann das Leben des Seins, oder das Leben Gottes oder des Geistes nicht beeinträchtigen. Mit dem Weg der „Verneinung des Nichts“- der Tod ist nicht Nichts - wehrt sich Rosenzweig gegen diese Lüge der philosophischen Tradition. Der Tod ist nicht nichts, und

${ }^{70}$ Diese zwei Wege setzen verschiedene Akzente. Rosenzweig schreibt, dass der Weg der Bejahung unendlich ist, der Weg der Verneinung aber endlich. Denn in der Antwort auf die Frage, was der Tod sei, ist das Feld nicht eingeschränkt, sondern potentiell „,unendlich“ breit, die Möglichkeiten, die geprüft werden wollen, sind erst mal potentiell unendlich. Dies ist bei der Frage „Ist der Tod nichts?" nicht der Fall, denn diese ist eine Ja-Nein-Frage, es stehen also potentiell nur zwei Antworten zur Verfügung. Rosenzweig schreibt: «Bejahung des Nichtnichts setzt - wie jede Bejahung, die durch Verneinung geschieht, - ein Unendliches, Verneinung des Nichts setzt wie jede Verneinung - ein Begrenztes, Endliches, Bestimmtes.» (GS II, 26)

${ }^{71}$ Rosenzweig stellt im Stern zuerst den Weg der „Bejahung des Nichtnichts“ dar und dann den Weg der „Verneinung des Nichts“. Hier kehren wir aus Klarheitsgründen die Ordnung der Darstellung um.

${ }^{72}$ GS II, 13.

${ }^{73}$ GS II, 13.

${ }^{74}$ GS II, 3-4.

${ }^{75}$ GS II, 4. 
die Welt ist kein All, sondern sie besteht aus vielen einzelnen Teilen, die eine gewisse gegenseitige Autonomie haben. Es gibt kein Leben ausserhalb dieser einzelnen Wesen. Ein All, das lebt, gibt es nicht. Diese ist die erste, ja vielleicht die wichtigste Erkenntnis, wodurch Rosenzweig den Begriff „Metalogik“ charakterisiert: ein Ganzes, das lebt, gibt es nicht, es gibt nur einzelne Teile bzw. Einzelwesen, die leben und die als Nebeneffekt durch die Beziehungen, die sie zueinander pflegen, evtl. ein Ganzes ausmachen ${ }^{76}$. Alles, was existiert, ist metalogisch ${ }^{77}$. Die einzelnen Teile leben und existieren, das Ganze nicht. Rosenzweig fügt hinzu: «dass die Philosophie das Einzelne aus der Welt schaffen muss, diese Ab-schaffung des Etwas ist auch der Grund, weshalb sie idealistisch sein muss» ${ }^{78}$.

Aber wieso wird dies, dass nur die Einzelnen leben und nicht das Ganze, als „metalogisch“ bezeichnet? Denn in jedem „idealistischen“ (im Sinne Rosenzweigs - d.h., dass die Ideen ein Leben haben bzw. lebendig sind) System hat das All eine bestimmte „logische“ Dynamik. Die einzelnen Wesen können im Rahmen dieses Alls „abgeleitet“ werden, die einzelnen Wesen verhalten sich „logisch“, weil das System „logisch“ gestaltet ist und diese Wesen im System fest verankert sind (Rosenzweig spricht von «Emanation» ${ }^{79}$ ). Wieso verhält sich dieses All „logisch“? Rosenzweig schreibt: «Worauf beruhte denn jene Allheit? Weshalb wurde denn die Welt nicht etwa als Vielheit gedeutet? Hier steckt offenbar eine Voraussetzung [... ]: die der Denkbarkeit der Welt. Es ist die Einheit des Denkens [...]. Die Einheit des Logos begründet die Einheit der Welt als eine Allheit. Und hinwiederum bewährt jene Einheit ihren Wahrheitswert in dem Begründen dieser Allheit.» ${ }^{80}$ Die Welt muss als Allheit gedacht werden, damit sie überhaupt denkbar sein kann, denn denken heisst, die Beziehungen zwischen den Elementen, die das Ganze ausmachen, zu verstehen. Wenn das Ganze verständlich ist, muss dieses Ganze sich nach „logischen Mustern“ verhalten und entwickeln (und dementsprechend die Einzelteile dieses Ganzen ebenso), meinen die Idealisten, da ich diese Beziehungen nachvollziehen kann. Die Philosophie setzt deswegen die Identität von Sein und Denken voraus, um die Übereinstimmung zwischen Sein und Denken zu begründen.

${ }^{76}$ Rosenzweig schreibt: «hier [beim Metalogischen] gilt das All nicht mehr als das eine und allgemeine, sondern als „ein“ All» (GS II, 50-51).

77 Der gesamte Stern ist metalogisch aufgebaut, es gibt nur Teile, die drei «Elemente» (GS II, 2) Gott (GS II, 25), Welt (GS II, 44) und Mensch (GS II, 67) die später (im zweiten Teil des Sternes) in Beziehung treten. Diese Beziehungen werden «Schöpfung» (GS II, 124), «Offenbarung» (GS II, 174) und «Erlösung» (GS II, 229) genannt.

${ }^{78}$ GS II, 4.

79 GS II, 55 (dieser Ausdruck ist aus der philosophischen Tradition übernommen, wie z.B. bei Plotin).

${ }^{80}$ GS II, 12-13. 
Aber für Rosenzweig gilt die «Nichtidentität von Sein und Denken ${ }^{81}[\ldots]$. Er fügt hinzu: «Da der Grund der Einheit von Sein und Denken im Denken gesucht wird [bzw. gesucht wurde, von den Idealisten], so müsste zunächst im Denken der Grund der Nichtidentität aufgedeckt werden $»^{82}$ und das ist es, was Rosenzweig mit dem Weg der "Verneinung des Nichts" macht, denn damit hat er den Allheitsgedanke kritisiert. Wie ist aber die Welt denkbar, wenn es keine Identität zwischen Denken und Sein gibt? Diese Frage wird anhand des Weges der „Bejahung des Nichtnichts“ beantwortet.

Die Welt ist ein All. Diese ist eben die grösste Scheinweisheit, die Rosenzweig verleugnen möchte: es gibt kein All, es gibt kein System! Die Welt ist nicht „logisch“, wie die Idealisten sie sich vorgestellt haben. Allerdings heisst das nicht, dass sie unlogisch sei, denn wir haben ja gesagt, dass wir eine Erkenntnis haben und diese Erkenntnis auch irgendwie „logisch“ ist (wir werden im Folgenden sehen, in welchem Sinne Rosenzweig hier von „Logik“ spricht). Die Welt ist nicht „unlogisch“, aber auf keinen Fall „logisch“ im Sinne des Idealismus: sie ist metalogisch, das heisst jenseits der Logik, weil das erste, der Ausgangspunkt, die Notwendige und unabdingbare Voraussetzung für die Existenz einer Welt, die Existenz des Einzelnen ist und nicht die des Alls. Das All des Idealismus mag logisch sein, es existiert für Rosenzweig nicht. Was existiert, sind die einzelnen Wesen und was wir über sie bis zu diesem Zeitpunkt der Argumentation behaupten können, wurde bereits dargestellt: sie sterben, sie sind sterblich.

Der Weg der „Verneinung des Nichts“ ist also sehr fruchtbar und unabdingbar für Rosenzweig. Allerdings konnten wir somit das Lebendige nicht positiv definieren. Dafür müssen wir den zweiten Weg beschreiten: den Weg der „Bejahung des Nichtnichts". Wir stellen uns nun die Frage, was denn der Tod sei.

\subsection{Die „Bejahung des Nichtnichts“}

Was ist der Tod? Ich weiss es nicht, ich weiss nur, dass man stirbt. Was stirbt also? Wenn ich die allgemeinste Antwort versuchen würde, die fast wie eine Tautologie klingen könnte, würde ich antworten: das Lebendige. Das Lebendige stirbt. Was ist aber das Lebendige? Kann ich das sagen? Ich kann behaupten, dass ich lebe. Ich lebe, ich existiere, ich bin. Um zu sterben, muss ich ja sein. Und weil der Tod mir Angst macht, weil ich sterben werde, heisst es, dass ich keine "quantité négligeable ${ }^{83}$ bin und dass ich bzw. meine existenzielle Situation des Leidens, der Angst und des Sterbens der Ausgangspunkt jedes meines Erkennens sein darf. Wenn ich lebe, stellt sich die Frage: Wer bin ich? Was bin ich? Was ist das Lebendige überhaupt? Wenn es eine Welt gibt, die aus verschiedenen lebendigen Wesen besteht, was sind diese Wesen? Wenn diese Wesen

\footnotetext{
${ }^{81}$ GS II, 13.

${ }^{82}$ GS II, 13; Anmerkung in Klammern von mir.

83 "Ich bin nicht unwichtig".
} 
sterben, wie kann die Welt weiter bestehen, wenn es diese Wesen sind, die die Welt ausmachen? Die Welt kann offenbar bestehen, weil andere Wesen geboren werden. Rosenzweig schreibt: «Ohne Aufhören gebiert Neues der Schoss der unermüdlichen Erde, und ein jedes ist dem Tode verfallen.» ${ }^{84}$ In diesem Satz ist bereits am Anfang des Sternes die Dynamik der Welt geschildert. Wir haben nun herausgefunden, was die Welt ist.

Kann ich mich aber somit zufriedengeben? Habe ich nun positiv bestimmt, was das Lebendige ist? Gibt es ein Wort, das diese Dynamik beschreibt, das für alle existierenden Wesen zutrifft, und das mir Erkenntnis, d.h. Ordnung in dieses Chaos der Welt bringen würde? Für Rosenzweig ist dies der Fall: der Begriff heisst "Gattung» ${ }^{85}$. Denn nur der Einzelne stirbt, die Gattung aber nicht, sie erneuert sich durch den Tod des einzelnen Wesens.

Dieser Schritt zur „Gattung“ ist deswegen sehr wichtig, weil er der entscheidende Schritt zum metalogischen Aspekt der Erkenntnis, zum Sinn der Welt ist. Mit diesem Begriff können wir die Dynamik der Welt feststellen: Die Gattung ist das Allgemeine, das über den Einzelnen steht. Wenn ich mich frage, was die Welt sei, muss ich feststellen, dass denken (in diesem Falle der Versuch, diese Frage nach der Welt zu beantworten), immer denken an etwas ist. Ich kann mir also dieses „etwas“ bzw. die vielen „etwas“, die einzelnen Wesen vorstellen, aber das sagt mir noch nichts darüber, was die Welt ist. Diese begrifflose Welt, die wir anhand der „Verneinung des Nichts“ gefunden haben, ist chaotisch für mich, erscheint mir ungeordnet, ich habe in diesem Sinne kaum Erkenntnis von ihr, mir fehlt die Orientierung. Um diese Welt begreifen zu können, brauche ich allgemeine Begriffe wie „Mensch, Tier, Staat, Bürger, usw.“, die die innersten Beziehungen zwischen den einzelnen Wesen aufweisen. Die Beziehungen, von denen im ersten Teil des Sternes die Rede ist, sind keine Beziehungen, die jeder Einzelne in seinem Leben bewusst pflegt oder vermeiden kann. Es sind die Beziehungen „des Seins“ der einzelnen Wesen.

Der Weg der „Bejahung des Nichtnichts“ ist bei Rosenzweig fruchtbar für den Sinn der Welt, für die Erkenntnis. Ich kann nur durch Begriffe, die eine Ordnung in die vor mir liegende und erscheinende Welt bringen, die Welt verstehen. Denn ohne die allgemeinen Begriffe der Vernunft, kann ich nichts über diese Anhäufung an besonderen Wesen sagen. Rosenzweig schreibt: «hier [...] beschreiben wir gewissermassen die ganze Kurve des Vorgangs. Der eine [...] Punkt ist der, wo nach einem Stück reinen, richtungs- und bewusstseinslos blinden Stürzens das Besondere sich seiner gezogenen Bewegung nach dem Allgemeinen hin gewissermassen bewusst wird und ihm dadurch die Augen über seine eigene Natur aufgehen. In diesem Augenblick wird das vorher blinde Besondere überhaupt zum seiner Besonderheit bewussten [...] Besonderen. Ein Besonderes, das vom Allgemeinen „weiss“, [...] [d] as ist das „Individuum“, das Einzelne, das die Merkmale des Allgemeinen, seiner Art, seiner Gattung, am

${ }^{84}$ GS II, 3.

${ }^{85}$ GS II, 52. 
Leibe trägt und trotzdem noch wesentlich Besonderes, aber nun eben „individuelles" Besonderes ist» ${ }^{86}$.

Rosenzweig fügt hinzu: «Es gibt einen Vorgang, worin sich diese beiden Elemente des Weltwesens [...] spiegeln. Das Individuum entspringt in der Geburt, der Genus [...] in der Begattung. Der Akt der Begattung geht der Geburt voraus und geschieht als einzelner Akt ohne die bestimmte Beziehung auf sie als Einzelne, dennoch in seinem allgemeinen Wesen streng auf sie bezogen und gerichtet. Die Geburt aber bricht nun in ihrem individuellen Ergebnis hervor [...]. Begattung gab es immer und dennoch ist jede Geburt etwas absolut Neues. Die Eigenheit des Geborenen [...] sammelt sich ganz im Augenblick der Geburt [...] insofern er Individualität, also ein besonderer Teil der Welt gleich jedem andren aussermenschliche Wesen oder Ding, ist» ${ }^{87}$.

Jedes einzelne Wesen ist also ein Individuum, es hat eine bestimmte Beziehung zum Allgemeinen, es trägt diese Beziehung ja sogar „am Leibe“, aber das, was lebt, sind die „Individuen“ und nicht die allgemeinen Begriffe selbst, wie z.B. Hegel behauptet. Der Beweis dafür ist, dass ein allgemeiner Begriff nicht sterben kann. Die Gattungen, die Begriffe, sind keine Subjekte, die leben. Was sind sie dann? Sie sind eben reine Begriffe, die man „wunderbarerweise“ auf die Welt anwenden kann. Rosenzweig schreibt: «Das Denken ist als ein vielverzweigtes System einzelner Bestimmungen in die Welt ergossen [...] Es verdankt seine Bedeutung für die Welt, seine „Anwendbarkeit“, jener Verzweigung, jener Vielfältigkeit, zu der es sich entschlossen hat» ${ }^{88}$. Die Begriffe der Vernunft sind „anwendbar“, „existieren“ aber nicht, nur das einzelne Wesen existiert. Deswegen gibt es lediglich Übereinstimmung zwischen Denken und Sein, und nicht Identität, wie der Idealismus behaupten würde, und das Sein, wenn man unbedingt davon sprechen möchte, wird vom Dasein, also von den einzelnen Wesen, abgeleitet, und nicht umgekehrt.

Nun können wir uns die Frage stellen, die wir vorher unbeantwortet gelassen haben: wie kann die Welt denkbar sein, wenn es keine Identität zwischen Sein und Denken gibt? Wie entsteht diese Übereinstimmung zwischen Denken und Sein, wenn nicht durch eine Identität zwischen Sein und Denken? Rosenzweig schreibt: «indem [...] [das Allgemeine] Anwendung verlangt, geht eine anziehende Kraft von ihm aus. So bildet sich um das Allgemeine ein Kraftfeld der Anziehung, in das das Besondere unter dem Zwange seiner eigenen Schwere hineinstürzt. ${ }^{89}$ Rosenzweig liefert uns also keine Begründung, wie das Denken auf die Welt angewendet werden kann ${ }^{90}$, keine Begründung dafür, wieso

${ }^{86}$ GS II, 51-52; kursiv von mir.

${ }^{87}$ GS II, 53.

${ }^{88}$ GS II, 46

${ }^{89}$ GS II, 51.

${ }^{90}$ Rosenzweig scheint hier eine Metapher aus dem Bereich der Chemie zu verwenden. Er stellt diese „,anziehende Kraft“ fest, ohne zu zeigen, wie sie entsteht. 
Welt und Logos übereinstimmen. In der Tat ist das für Rosenzweig nicht zu begründen ${ }^{91}$, denn: «[d]as bloss vorausgesetzte Denken mag gedacht werden müssen, denkt aber nicht; bloss das wirkliche, das weltgültige, weltangewandte, weltheimische Denken denkt. So bleibt die Einheit des Denkens ausserhalb; das Denken muss sich dafür trösten mit der Einheit der Anwendung in den geschlossenen Mauern der Welt. ${ }^{92}$ Was sind Begriffe? Was sind Namen? Sie bestehen in der reinen Möglichkeit ihrer Anwendung auf die Welt und das ist das, was Rosenzweig mit «Logos» ${ }^{93}$ meint. Er schreibt: «Der Logos ist das Wesen der Welt ${ }^{94}$. Die Welt ist also metalogisch: sie existiert jenseits und unabhängig von der Logik; die Begriffe, der Logos, „,existieren“ nicht (sie haben kein eigenes Leben). Die Logik besteht nur darin, auf die Welt angewendet werden zu können und deswegen ist diese Welt nicht unlogisch, sondern metalogisch. Denken bedeutet, anhand der Sprache denken. Rosenzweig spricht von «Sprachvernunft»" . Ohne Sprache keine Vernunft. Ohne Vernunft kein Denken. Ohne Denken kein Sinn der Welt.

\subsection{Mathematische Zeichen}

Der Logos, das Denken in Begriffen, ist für uns das Wesen der Welt. Die Welt ist begreifbar. Danach suchen wir, wenn wir uns die Frage stellen, was die Welt sei, denn wir versuchen sie zu begreifen. Was die Welt ist, diese „was-ist-Frage“, kann nur unsere Erkenntnis beantworten: „Die Welt ist... “ und das, was folgt, sind notwendigerweise Begriffe. Allerdings ist das Wesen der Welt „dynamisch“ und nicht statisch. Wir haben bereits gesehen, dass «[j]edes Besondere [...] bei seiner Geburt [...] nichts als seiend [ist]. Seine Kraft ist nur das blinde Schwergewicht seines Seins» ${ }^{96}$ und dass «die logischen Formen [...] das Unbewegte, das „ewig Gestrige“, das „Allgemeine“ [sind]»" Allerdings «in der Bewegung, die das Individuum in die geöffneten Arme der Gattung hineinführt, vollendet sich die Gestalt der Welt.» ${ }^{98}$

Um diese Bewegung innerhalb des Seins der Welt zu beschreiben, bedient sich Rosenzweig der Symbole der Mathematik, denen er aber eine völlig

${ }^{91}$ Eine weitere Passage des Sternes, die dies zu betonen scheint, ist die folgende: «Diese seine [jedes Weltteils] Individualität wird nun mit dunkler Gewalt angezogen von der Macht ihrer Gattung» (GS II, 53-54; kursiv von mir), „dunkel“, weil nicht zu erklären.

92 GS II, 46-47.

${ }^{93}$ GS II, 46.

${ }^{94}$ GS II, 46; kursiv von mir.

95 GS II, 36.

${ }^{96}$ GS II, 49.

97 GS II, 50.

98 GS II, 52; kursiv von mir. 
eigenartige Bedeutung verleiht, die mit der üblichen Mathematik, die wir aus der Schule kennen, wenig zu tun hat.

Rosenzweig beschreibt den Logos als, $\mathrm{B}=\mathrm{A}^{(499}$, also „Besonders=Allgemein ${ }^{“ 100}$. Was „A“ betrifft, schreibt er: "[w]ollen wir für diesen schlechthin allgemeinen und doch überall der Welt verhafteten, in sie eingebundenen Logos eine formelhafte Bezeichnung, so müssten wir ihn als Ergebnis einer Bejahung auf der rechten Gleichungsseite auftreten lassen; um seiner Allgemeinheit willen, die keinen Raum neben sich freilässt, dürften wir ihn nur mit A bezeichnen ${ }^{101}$. Das Zeichen „=“ beschreibt das Merkmal der Anwendbarkeit der Allgemeinheit der Begriffe auf die Welt, ja die Anziehungskraft, die diese den Einzelwesen gegenüber haben: «der Charakter der Anwendbarkeit, den wir ihm als wesentlich erkannten, bedeutet einen Hinweis auf die Notwendigkeit, dass die Anwendung an ihm auch wirklich geschehe; diese passiv anziehende Kraft, die von ihm ausgeht, wurde im Symbol ausgedrückt durch den Vorantritt des Gleichheitszeichens. So erhalten wir “ $=A^{“} .{ }^{102}$ Was „B“ betrifft, schreibt er: "Jedes Besondere [...] bei seiner Geburt ist es blind, es ist nichts als seiend. Seine Kraft ist nur das blinde Schwergewicht seines Seins. Das Symbol nach unsrer Terminologie ist B, schlechtweg B, das nackte Zeichen der Einzelheit, ohne ein hinweisendes Zeichen der Gleichheit.» ${ }^{103}$ Die Welt hat diese dynamische Bewegung in sich, «ihr Schoss ist nimmersatt zu empfangen, unerschöpflich zu gebären. Oder besser - denn Männliches und Weibliches ist beides in $\mathrm{ihr}$ - sie ist als „Natur" ebenso die endlose Gebärerin der Gestalten wie die nie ermattende Zeugungskraft des in ihr heimischen "Geistes“.»"104 "So entsteht die Gestalt der Welt, [...] aus dem Eingehen des Individuums in die Gattung.» ${ }^{105}$

Rosenzweig verwendet nicht nur mathematische Zeichen, sondern auch ein bestimmtes Fachvokabular, um diese Dynamik der Welt zu beschreiben. Diese Dynamik, genannt die «Wirklichkeit der Welt»" ${ }^{106}$, entsteht aus der Synergie zwischen dem allgemeinen und besonderen Charakter der Welt, also zwischen jeweils der «weltliche[n] Ordnung» ${ }^{107}$ und der "weltliche[n] Fülle» ${ }^{108}$.

Eine letzte Frage bleibt noch ungeklärt: was ist der Tod (denn diese war unsere Ausgangsfrage)? Der Tod kann für Rosenzweig auf diesem Stadium des Sternes nur innerhalb dieser Dynamik der Welt verstanden werden.




Andere Aspekte dieses Teils des Sternes, wie z.B. die griechische Welt, die orientalische Welt und die Kunst können hier nicht behandelt werden. Es sei nur Folgendes gesagt: Rosenzweig zeigt, dass die griechische Welt bereits metalogisch gedacht hat und die indische bzw. chinesische Welt jeweils einen Aspekt des Metalogischen (die indische das Besondere und die chinesische das Allgemeine) in ihrer Denkweise unterschätzt haben. Ob dies philologisch bzw. philosophisch korrekt sei, kann hier auf keinen Fall besprochen werden.

In Bezug auf das Thema der Kunst: die Ausführungen Rosenzweigs über die Kunst sind überall im Stern „systematisch verstreut" und sind im Einzelnen nur schwer zu verstehen. Diese können nur im Gesamtbild des Sternes nach einer sorgfältigen Rekonstruktion verstanden werden. ${ }^{109}$

\section{Jenseits der Philosophie}

Sie können sich nun fragen, ob das Leben, ob die Welt wirklich nur eine reine Anhäufung an Einzelwesen ist, die Gott sei Dank verstanden werden können. Laut Rosenzweig lautet die Antwort: nein. Die Unzulänglichkeit dieser bisher geführten Argumentation ist der philosophischen Haltung zu schulden. Der Philosoph fragt „Was ist?“, und diese „Was-ist-Frage“ ist bereits der Fehler für Rosenzweig, denn diese Frage will eine ewige Antwort haben; die Philosophie will ewige Antworten, und dies ist eben verwerflich, denn all das, was existiert, ist nicht ewig, sondern steht unter der Knute der Zeit.

Diese ist die ganze Spannung, die es zwischen gesundem Menschenverstand und der Philosophie gibt, laut Rosenzweig. Rosenzweig schreibt: «Der gesunde Menschenverstand steht im Verruf bei den Philosophen. Er soll wohl dazu genügen, ein Viertelpfund Käse einzukaufen, einen Heiratsantrag zu machen, [...] - aber die Antwort auf die Frage, was der Käse, die Frau [...] eigentlich sei, diese Antwort dürfe man von ihm nicht erwarten, hier habe der Philosoph einzutreten ${ }^{110}$.

Was ist eigentlich der Tod? Was ist eigentlich die Welt? Eigentlich bedeutet: hier wird keine einfache, schlichte Antwort verlangt, die vielleicht in zwei Monaten nicht mehr wahr ist, sondern eine, die ewig ist. Dieses Wort „eigentlich“ setzt voraus, dass die Welt doch etwas Anderes ist als das, was sie erscheint ${ }^{111}$. «So

109 Diese Arbeit wurde sehr akkurat und sorgfältig im folgenden Aufsatz geleistet: F.P. Ciglia, Kunst als Propädeutik der Erlösung. Ästhetik und Theologie im Denken Franz Rosenzweigs, in: M. Brasser (Hg.), Rosenzweig Jahrbuch 1/ Rosenzweig Yearbook 1, Rosenzweig heute / Rosenzweig today, Freiburg/München 2006, 134-154.

110 BGK, 28; kursiv von mir.

111 Die ganze Erkenntnistheorie der abendländischen Philosophie, ausgehend spätestens von Platon, fusst darauf: denn sie können nicht behaupten, ein Mensch sei ein Wesen mit zwei Beinen. Sind Menschen die ohne ein Bein geboren werden also keine Menschen? Doch. Also ist dies nicht relevant 
bleibt ihm [dem Philosophen] nun nichts anderes übrig als [...] sich einzubohren in das Problem, in den aus dem Fluss des Lebens herausgenommenen [...] „Gegenstand“ des Denkens. [...] Er fragt: was ist. [...] Der künstlichen Zeitlosigkeit der "Was-ist“-Frage antwortet die [...] Antwort: „Das Wesen.“ [...] Im Leben gilt diese Frage so wenig, wie dort die Frage vorkommt. Auch der Philosoph wird sie im Ernstfall nicht stellen. Er wird nicht fragen, was das Viertelpfund Käse „eigentlich“ kostet. Er wird seine Erkorene nicht fragen, ob sie „eigentlich“ seine Frau werden möchte. [...] Nicht „eigentlich“, sondern „wirklich“ ist das Wort des Lebens [...]. Hier [...] trennen sich seine Wege von den Wegen des gesunden Menschenverstands. Der gesunde Menschenverstand vertraut dem Wirklichen und seinem Wirken.» ${ }^{112}$ Aber $«[\ldots]$ ins eine All der Wirklichkeit trägt uns nur der eine Strom der Weltzeit» ${ }^{113}$.

Die Unzulänglichkeit der philosophischen Perspektive, die sogar jeglichen gesunden Menschenverstand brüskiert, sollte uns dazu bringen, diese Perspektive zu verlassen und den zweiten und dritten Teil des Sternes zu lesen, in der diese philosophische Perspektive eben überwunden wird.

Achtung: dies heisst nicht, dass die bis jetzt erbrachte Mühe umsonst war. Der Stern, die Wirklichkeit, sind metalogisch aufgebaut und die Angst vor dem Tod erfährt jeder. Ein Erkenntnisbewusstsein zu erlangen, ist nötige Voraussetzung des wahren Lebens. Der gesamte Stern kann wie ein Weg betrachtet werden, der gegangen werden muss, um diesen dann zu verlassen, wenn man am Ziel angekommen ist. Wie die wittgensteinische Leiter, die gebraucht wird, um aufzusteigen und dann aber eben zur Seite gelegt zu werden, können wir auf den Stern erst dann verzichten, wenn wir mit Rosenzweig den Weg «[v]om Tode» ${ }^{114}$ «ins Leben» ${ }^{115}$ gegangen sind.

\section{Weiterführende Lektüre}

Bertolino, L., (2000). La Filosofia del Nulla in Franz Rosenzweig, in: Annuario Filosofico 16 (2000) 257-287; [https://philpapers.org/archive/BERLFD-10 .pdf]

Losch, A., (2011), Der Stern, im Licht der Offenbarung betrachtet - zur geometrischen Konstruktion des Sterns der Erlösung, in: Naharaim 5 (2011) 36-54. [https://www.degruyter.com/document/doi/10.1515/naha.2011.005/html]

für die Definition des Begriffes „Menschen“. Daher die Suche nach dem „Wesen“ des Menschen (im zweiten Buch des ersten Teils des Sternes stellt sich Rosenzweig die Frage nach dem Wesen der Welt), d.h. einer Antwort, die ewig ist.

112 BGK, 30-32.

113 GS II, 95; kursiv von mir.

114 GS II, 3.

${ }^{115}$ GS II, 472. 